ZAMM · Z. Angew. Math. Mech. 79 (1999) 6, 389-397

Vielsack, P.; Hartung, A.

\title{
An Example for the Orbital Stability of Permanently Disturbed Non-smooth Motions
}

\begin{abstract}
Die mathematische Modellierung eines mechanischen Systems mit Reibung und Stoß führt zur Problematik der Integration von Systemen mit veränderlicher Struktur. Von Interesse ist der Einfluß permanenter Störungen (sowohl physikalisch wie numerisch) auf die orbitale Stabilität der ungestörten Lösung. Hierbei zeigt es sich, daß beide Arten von Störungen sich in ähnlicher Weise auswirken und daß abhängig vom Typ der Lösung unterschiedliche Stabilitätsgrenzen existieren.
\end{abstract}

Developing a mathematical model of a mechanical system with friction and impact leads to problems regarding the integration of systems with variable structure. The main point of interest is the influence of permanent disturbances (both of physical and numerical nature) on the orbital stability of the non-disturbed solution. It is shown that both types of disturbances have similar effects and that, depending on the type of solution, different stability limits exist.

MSC (1991): 73T05, 65L07, 34D10

\section{Introduction}

Comprehensive literature on the subject of non-smooth dynamical systems has been made available in the last years [1]. Calculating the motion, we have to face two problems which can be treated separately. The first one is the integration of motion equations with a smooth right side between two switching points. The second one is the determination of the switching times and the choice of the correct switching decision. In both cases, the numerical calculations have only a limited degree of accuracy. Due to the nonlinearity of all contact problems, this is the primary source of errors, and it seems rather difficult to present a general theory. Such problems can only be treated on the basis of given examples. On the other hand, it is certainly possible to generalize qualitative properties. The basic idea is to presuppose that a nondisturbed problem and its solution are known and to make a comparison with a disturbed problem. The permanent disturbances can either be of physical nature $g(t, X)$ or may be caused by incorrect switching conditions or decisions. For the case of numerical disturbances, the definition of the undisturbed problem is relative. The issue is to make a comparison with a solution calculated as exactly as possible. The two types of disturbances are treated separately. The practically orbital stability according to LASALLE and LEFSCHETZ (cf. HAHN [2]) is extended to non-smooth systems.

The definition is as follows: The solution $X\left(t, X_{0}, t_{0}\right)$ of the undisturbed motion is called practically stable under persistent physical disturbances $g$ (numerical disturbances $\Delta t$ ), if for a number $k_{3}>0$, depending on the particular physical situation (the requirements of accuracy), there exist two positive numbers $k_{1}\left(k_{3}\right)$ and $k_{2}\left(k_{3}\right)$ such that

- for all $t \geq t_{0}$

- provided that for all $X_{0}^{1}, t_{0}^{1}: \quad|| X_{0}-X_{0}^{1} \|+\left|t_{0}-t_{0}^{1}\right|<k_{1}$ and for all $\mathrm{g}$ (or $\Delta t):\|g\|<k_{2}\left(\right.$ or $\left.\Delta t<k_{2}\right)$,

the disturbed solution $X^{1}\left(t, X_{0}^{1}, t_{0}^{1}\right)$ admits an estimate by the inequality

$$
\varrho\left(X\left(t, X_{0}, t_{0}\right), X^{1}\left(t, X_{0}^{1}, t_{0}^{1}\right)\right)<k_{3} .
$$

Instead of presuming explicit numbers $k_{3}$, in the following, we search for the limit of the number $k_{2}$, above which the solutions of the disturbed system no longer have the orbital structure of the solution $X\left(t, X_{0}, t_{0}\right)$. This means that subsequently the existence of structural stability according to GuCKEnHEIMER and HoLmES [3] is taken as a criterion for practically orbital stability of non-smooth motions.

\section{Mechanical model}

Consider a mass $m$ on an inclined plane (angle $\alpha$ ). For the state of sliding Coulomb's law (friction coefficient $\mu$ ) is presupposed. The relative coordinate $x$ gives the distance of the mass from an obstacle fixed at the plane. The plane moves with a known translational motion $y(t)$. It consists of a harmonic part (amplitude $a_{0}$, angular frequency $\Omega_{0}$ ) and permanent small perturbations. If the mass sticks, the adhesive force $F_{H}$ between mass and inclined plane is calculated as a passive force.

Introducing the dimensionless quantities $\tau=\Omega_{0} t,{ }^{\prime}=\mathrm{d} / \mathrm{d} \tau, \xi=\left(\Omega_{0}^{2} x\right) /(\mu g \cos \alpha), H=F_{H} /(m \mu g \cos \alpha), A=$ $\left(\Omega_{0}^{2} a_{0}\right) /(\mu g \cos \alpha), B=\tan \alpha / \mu$ the motion equation for sliding reads

$$
\xi^{\prime \prime}+\operatorname{sgn} \xi^{\prime}=A[\sin \tau+f(\tau)]-B, \quad\left|\xi^{\prime}\right|>0, \quad \xi>0, \quad \tau_{i}<\tau \leq \tau_{i+1} .
$$




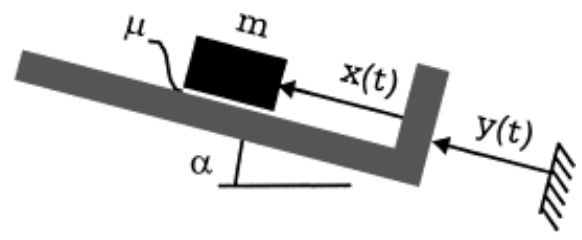

Fig. 1. Mechanical model

The function $f(t)$ describes permanent small perturbations. During an interruption the adhesive force is given by the algebraic equation

$$
H=A[\sin \tau+f(\tau)]-B, \quad|H|<1, \quad \tau_{i}<\tau \leq \tau_{i+1},
$$

and the state variables are known as

$$
\xi^{\prime}(\tau) \equiv 0, \quad \xi(\tau)=\xi_{0} \geq 0, \quad t_{i}<t<t_{i+1} .
$$

The equality corresponds to the interruption at the obstacle, the inequality to a state of sticking in a distance $\xi_{0}$ from the obstacle.

Five different states of the system can exist during the motion of the mass: relative sliding upwards or downwards, sticking far from the obstacle, interruption at the obstacle, and the impact at the obstacle. The latter is assumed to be ideal plastic. Each state begins at a switching time $\tau_{i}$ and ends at $\tau_{i+1}$. In the case of impact both times coincide. Presupposing the respective switching time $\tau_{i}$ to be known, every state can be treated separately. Especially the general solution of (1) reads

$$
\begin{array}{ll}
\xi_{+}=c_{1} \tau+c_{0}-\frac{1+B}{2} \tau^{2}-A \sin \tau+\xi_{p}, & \xi^{\prime}>0, \\
\xi_{-}=\bar{c}_{1} \tau+\bar{c}_{0}+\frac{1-B}{2} \tau^{2}-A \sin \tau+\xi_{p}, & \xi^{\prime}<0 .
\end{array}
$$

Without any restriction to the generality it can be assumed that the particular solution $\xi_{p}$ of the function $f(\tau)$ is known. The initial conditions for each state follow for $\tau=\tau_{i}$ from the known values of the preceding state. This gives

$$
\xi\left(\tau_{i}+0\right)=\xi\left(\tau_{i}-0\right), \quad \xi^{\prime}\left(\tau_{i}+0\right)=\xi^{\prime}\left(\tau_{i}-0\right),
$$

and

$$
\xi\left(\tau_{i}+0\right)=\xi^{\prime}\left(\tau_{i}\right)=0
$$

in the case of impact. A special application of the problem in consideration is the most simple approximation of a technical process, the so-called vibratory driving of piles into granular soil by harmonic excitation [4], [5].

\section{The integration method and the numerical problem}

The total solution is achieved by combining the five possible states in an order given by the respective previous history. This means that for a known switching time $\tau_{i}, i=0,1,2, \ldots$, the new $\tau_{i+1}$ has to be determined. The switching time $\tau_{0}$ characterizes the very beginning of the motion. The appropriate initial conditions can be given arbitrarily. However, in contrast to smooth dynamics, a consistent system state has to be chosen. The complete facts are shown in the scheme of Fig. 2. Arrows indicate the change from the previous state to the following one.

Between two states, we first have the switching condition and then the switching decision. For impact, the switching condition for the next state becomes void, as this is an instantaneous process. For interruption at the obstacle and for transition from a motion with $\xi^{\prime}<0$ to impact, the switching decision becomes void. All five switching decisions were formulated as test equations. They are as follows:

$$
\begin{array}{ll}
S_{1}=\xi_{-} ; & \tau>\tau_{i}, \\
S_{2}=\xi_{-}^{\prime} ; & \tau>\tau_{i}, \\
S_{3}=\xi_{-}^{\prime} ; & \tau>\tau_{i}, \\
S_{4}=|H|-1 ; & \tau>\tau_{i}, \\
S_{5}=H-1 ; & \tau>\tau_{i} .
\end{array}
$$

The switching decisions are regulated by check functions. The scheme shows that the adhesive force $H$ is important for the decision only. At this stage, the authors point out that according to equation (2) $H$ contains the disturbances. The inaccurate determination of a switching point may therefore lead to wrong decisions.

To determine the end of a state, up to two test functions have to be calculated for each time. The first zero of a test function in the open interval $\tau>\tau_{i}$ is the switching time $\tau_{i+1}$. The zeros can be determined with limited accuracy 


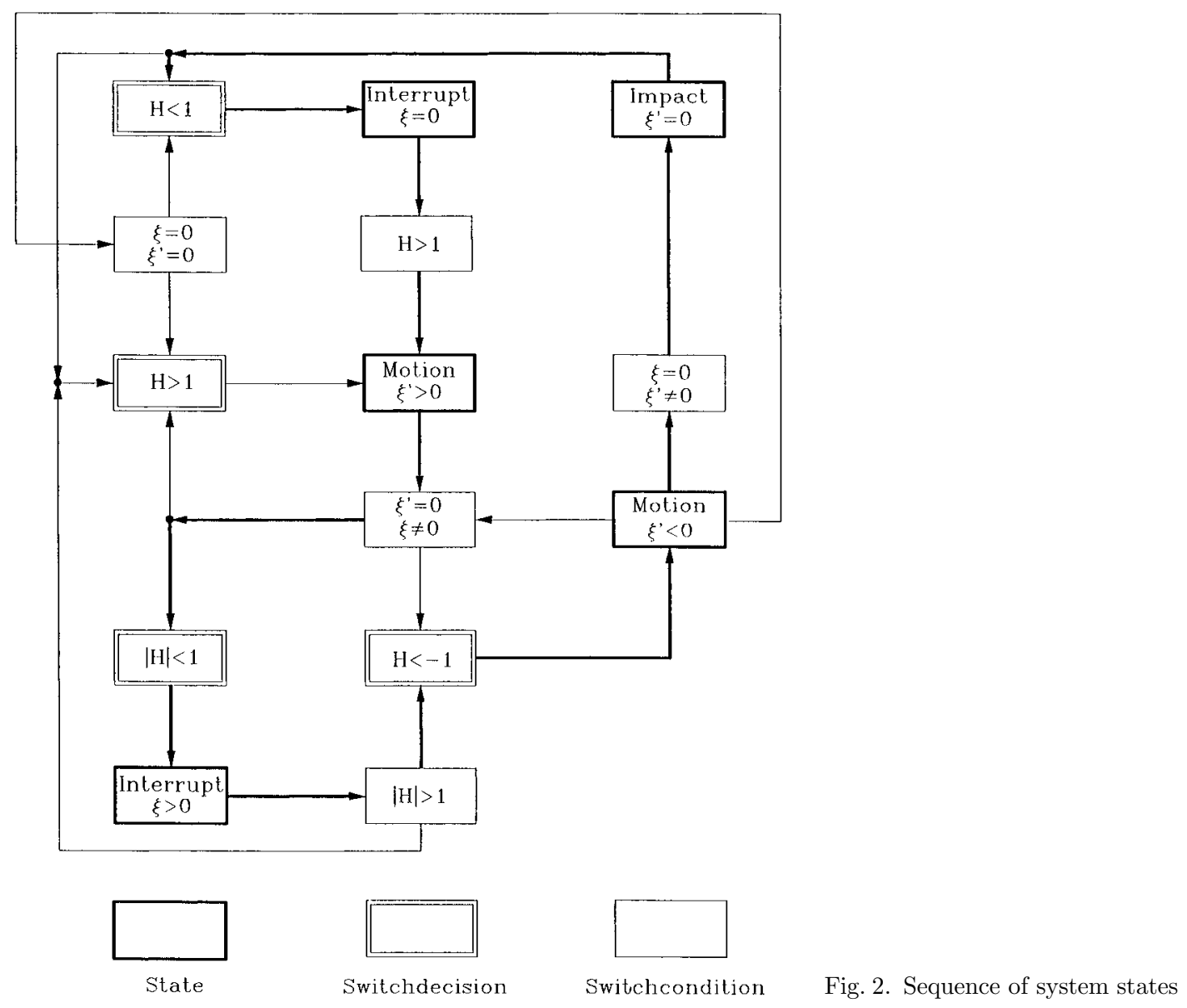

only. Problems occur in the case of multiple zeros, which has already been indicated in [6]. Another problem is to recognize two coinciding zeros of two different test functions, as is the case when the mass touches the obstacle without impact.

Depending on the two parameters $A$ and $B$ of the non-disturbed problem, the scheme is passed through in a certain order. Due to the periodicity of the non-disturbed excitation, periodic solutions are expected. In reality, bifurcations up to chaos can exist. The most simple type of solution, however, is the sequence marked in the scheme by thick arrows.

First iterative statements for periodic solutions of non-smooth systems are given in [7]. [8] gives an algorithm for the localization of zeros. The advantage of the investigated mechanical model is that for a known particular integral $\xi_{p}$ in the equations (4) and (5) closed solutions of the motion equations in the form of algebraic expressions can be given. In this case, a numerical integration is not required. The total problem can be expressed in algebraic terms. The numerical problem is reduced to the determination of zeros of algebraic equations, i.e. of the switching functions (8). The choice of a step size for a numerical integration now corresponds to the selection of a small time interval $\Delta \tau$, which is supposed to contain a switching point. It becomes evident that the selection of such an interval has to be assessed in the same way as the choice of a time step size for numerical integration in the case of non-smooth systems: The choice of $\Delta \tau$ is merely heuristic. This is due to the fact that it is not known a priori how many zeros occur in an interval $\Delta \tau$ during the motion $\tau>\tau_{i}$. If, in this case, several roots of the currently valid switching function exist, the smallest one characterizes the switching point. The numerical problems have been discussed in [8]. In the following, some modifications are introduced. This results from the fact that no numerical integration is necessary. Even here, it becomes obvious that most errors in calculating the total motion result from the numerical inaccuracy of the determination of the switching times. A wrong localization of the switching points leads to wrong state variables $\xi$ and $\xi^{\prime}$. Wrong state variables at a switching point correspond to instantaneous coordinate disturbances for $\xi$ and $\xi^{\prime}$ with respect to the non-disturbed problem. These disturbances are permanently found at all switching points.

\section{Permanent physical disturbances}

Without any restriction to the generality, we assume $B=0.1$ in the following. The type of solution then only depends on the parameter $A$. The bifurcation diagram (non-disturbed problem) for the velocity $\xi^{\prime}$ in dependence on $A$ is shown in Fig. 3. It is similar to that of the logistic equation. 


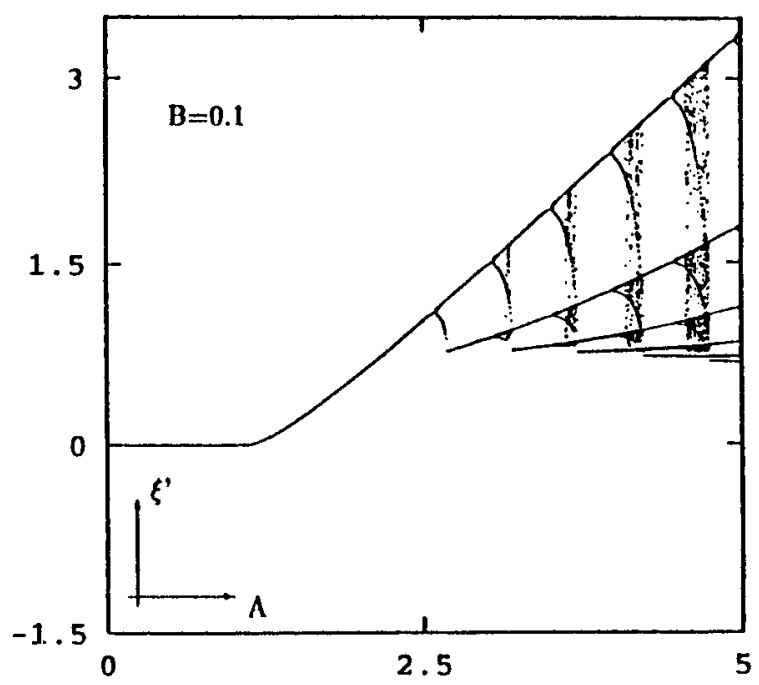

Fig. 3. Bifurcation diagram of the non-disturbed problem

The way to chaos is clearly visible. However, the investigations were restricted to the beginning of the bifurcations, i.e., in the following $0<A<3.25$.

The bifurcation diagram is calculated for each parameter value $A \leq 3.25$ on the basis of transient vibrations. However, this method does not supply information on unstable solutions of the undisturbed motion. The initial conditions may be chosen arbitrarily. They lead to asymptotically stable orbits. Nevertheless, the motions are not necessarily unique. The structure of the solution varies as the number of bifurcations increases. The $n$-th subharmonic motions consist of $n$ (according to LJAPUNOV) asymptotically stable solutions, which have the same shape but are shifted against each other by the phase $2 \pi$ (dimensionless excitation period). The most simple case is illustrated in Fig. 4 , which shows for $A=3.04$ the velocity $\xi^{\prime}$ after 1100 excitation periods during $6 \times 2 \pi$ in comparison with the harmonic excitation.

Different initial conditions lead to different phase positions in relation to the excitation. Since the phase shifting between different non-unique solutions is always $2 \pi$ for equal parameter values, this special structure is not visible in the bifurcation diagram and in phase curves.

Perfectly harmonic driving mechanisms do not exist in real technical systems. Particularly with regard to the accelerations the excitation signals are very noisy. As an example we consider only a special disturbance with the particular solution

$$
\xi_{p}=-\varepsilon A \sin (\eta \tau)
$$

with small relative amplitude $\varepsilon \ll 1$ and high frequency ratio $\eta \gg 1$. Without any restriction to the generality we assume $\eta=10 \cdot \sqrt{102}$ for all following calculations. The irrational and thus incommensurable frequency ratio is chosen to avoid that a common periodicity of non-disturbed excitation and disturbation is introduced into the problem. A rational relation would cause a periodic solution with a large multiple of the excitation period and would thus be a special case. The magnitude of the disturbance is defined by the relative amplitude $\varepsilon$ only. For the non-
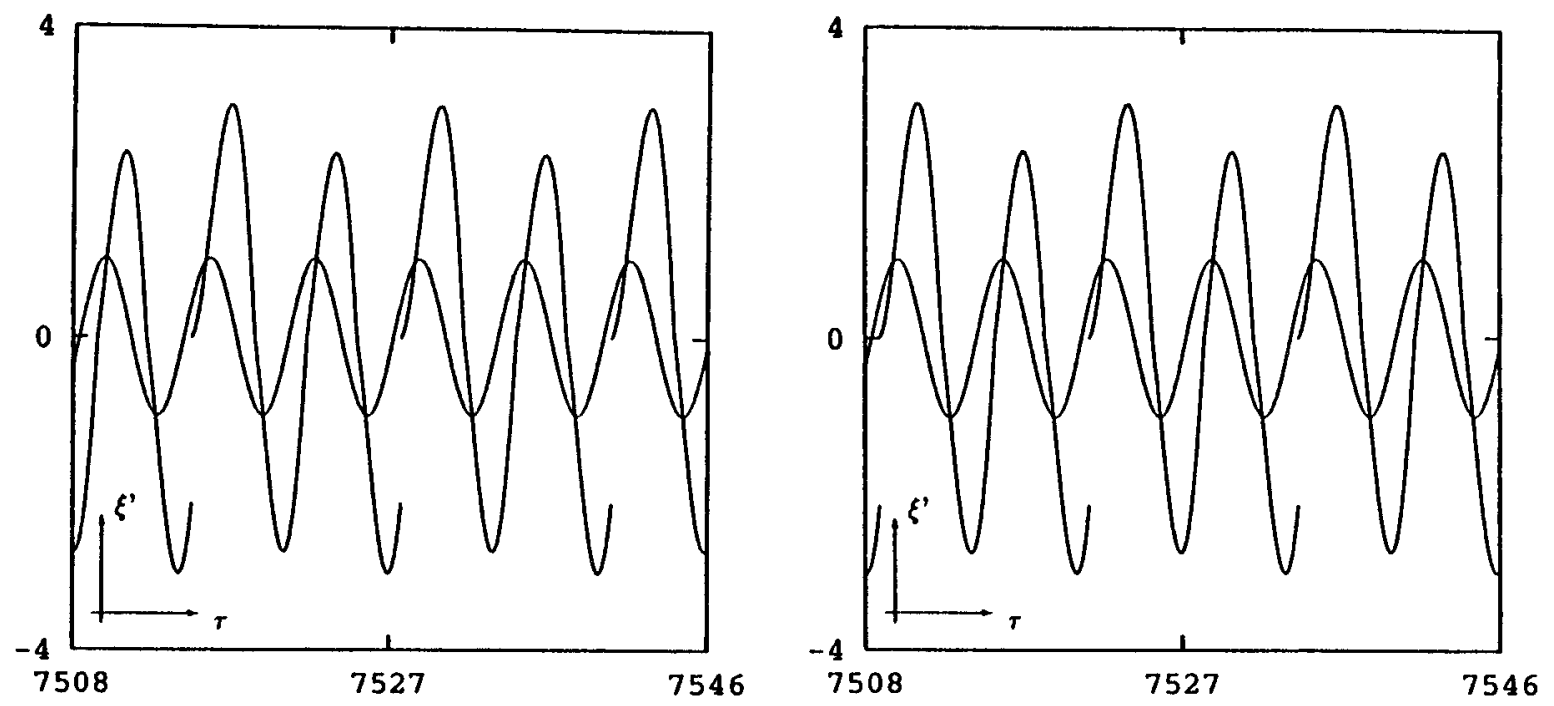

Fig. 4. Non-unique solutions $(A=3.04, B=0.1)$ with period $2 \times 2 \pi$ 

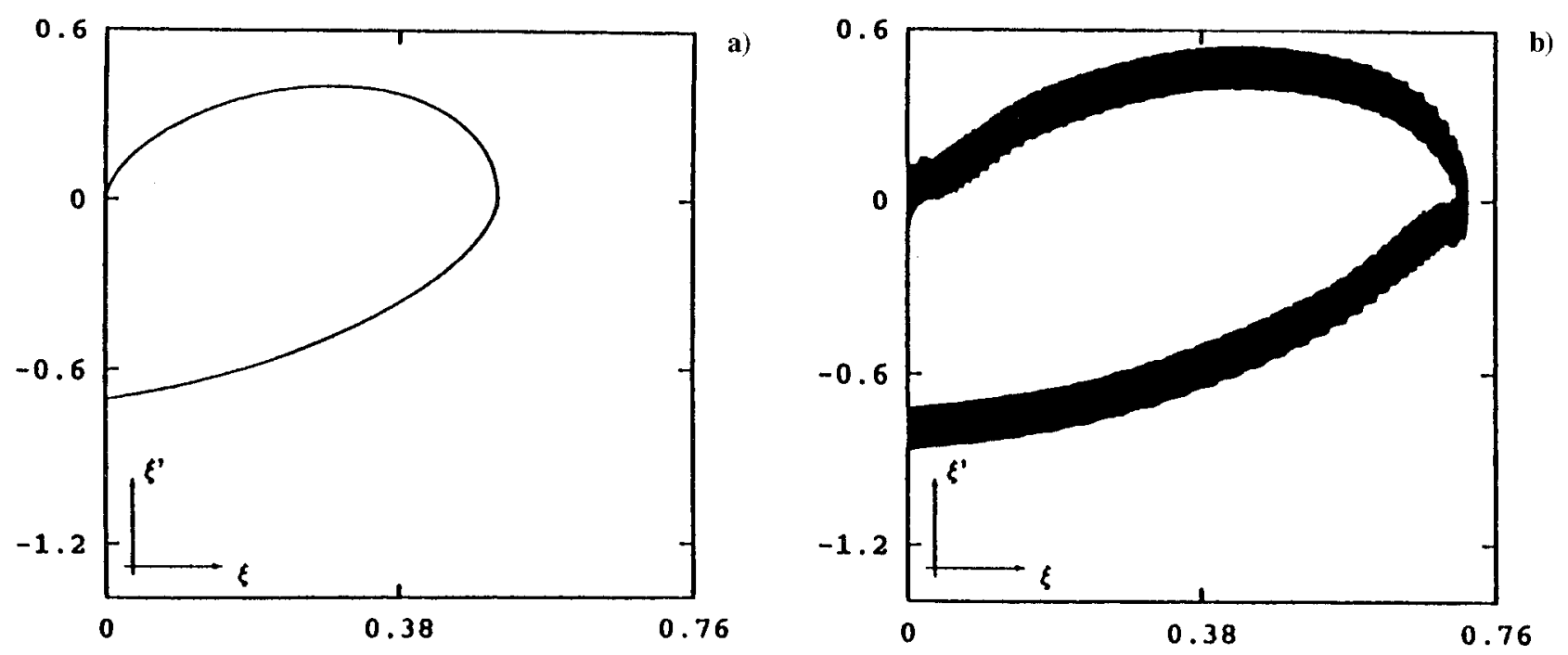

Fig. 5. Non-bifurcated solution, $A=1.5, B=0.1$ : a) non-disturbed; b) $\varepsilon=5 \cdot 10^{-4}$

bifurcated solution with $A=1.5$, Fig. 5 compares the non-disturbed result with a disturbed one. For this purpose, first 1000 excitation periods were calculated in order to eliminate transient vibrations. The figure shows the subsequent 250 periods.

Even if the amplitude $\varepsilon$ of the disturbance is relatively large, the structure of the non-disturbed solution is maintained. Indeed, the state quantities $\xi$ and $\xi^{\prime}$ in a response period vary from period to period (broad band in Fig. 5), altogether, however, we have a quasi-stationary motion with the same sequence of system states as in the case of the non-disturbed problem. The orbital stability is due to the existence of two interruptions (when the motion changes from $\xi^{\prime}>0$ to $\xi^{\prime}<0$ and at the obstacle) as well as to the influence of the plastic impact itself. As the excitation amplitude $A$ is increased, first the interruption at the return of the motion vanishes. The interruption after impact may be omitted partially or completely in the case of bifurcated solution. The investigation of the orbital stability is more complicated. The first subharmonic solution for $A=3.04$ is examined exemplarily by means of Poincaré sections using the results for 1500 excitation periods after 1000 periods of transient vibration (Fig. 6).

With regard to the non-disturbed problem, we obtain two points for the stationary solution. Small disturbances with $\varepsilon=0.5 \cdot 10^{-4}$ have the same course of motion, however, the amplitudes of $\xi$ and $\xi^{\prime}$ vary slightly due to quasistationary effects. If $\varepsilon$ increases further, the two points of the solution diverge. The stability limit is shown in Fig. $6 \mathrm{c}$. Larger values of $\varepsilon$ give motions with different structures. Graphically, this leads to a point cloud.

For further discussion, it is sufficient to consider exemplarily the range $3.0<A<3.25$ of the bifurcation diagram, where bifurcations and higher subharmonic solutions occur (Fig. 7). Practically orbitally stable solutions of the non-disturbed problem can be recognized as quasi-stationary motions by the structure, i.e., lines in the non-disturbed problem expand to black bands in the disturbed problem. 500 excitation periods after 500 transient periods were scanned. Thus, the density is not necessarily complete.

Subharmonic solutions of low order are orbitally stable in the case of sufficiently small disturbances. Instability effects particularly occur at points of bifurcations and in the case of subharmonic solutions of higher order. This result is confirmed by known experimental investigations of real mechanical systems [5]. The experimental verification of the first subharmonic motions in the bifurcation diagram according to Fig. 3 can be carried out easily. In the case of larger excitation amplitudes irregular motions occur in the test. The requirements on the numerical accuracy when calculating the disturbed solutions in Fig. 7 are extremely high. According to numerical investigations, the step size has to be $\Delta \tau=10^{-5}$. Furthermore, the switching times were additionally re-iterated to an accuracy of $10^{-7}$ following the MULLER procedure [9]. The required computation time, however, is justifiable for exemplary calculations only.

\section{Permanent numerical disturbances}

The required computation time described above can be avoided by using constant step sizes $\Delta \tau$. The switching points are determined with a limited degree of accuracy only. The switching functions are merely used to check whether the sign changes during the interval $(\tau, \tau+\Delta \tau)$. This corresponds to the condition

$$
S_{i}(\tau) \cdot S_{i}(\tau+\Delta \tau)<0
$$

where $S_{i}$ is one of the test functions in equations (8). If this is the case, the time $\tau+\Delta \tau$ is considered to be the switching time. This leads to permanent numerical disturbances in the course of the motion. In addition, the switching decision is not necessarily correct. The described procedure is applied in most calculation methods in contact mechanics. In the 


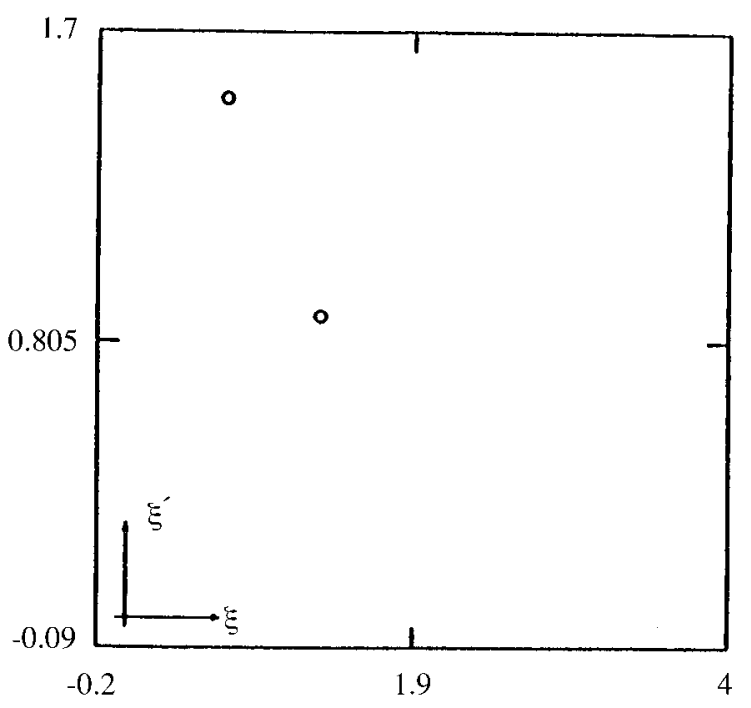

(a)

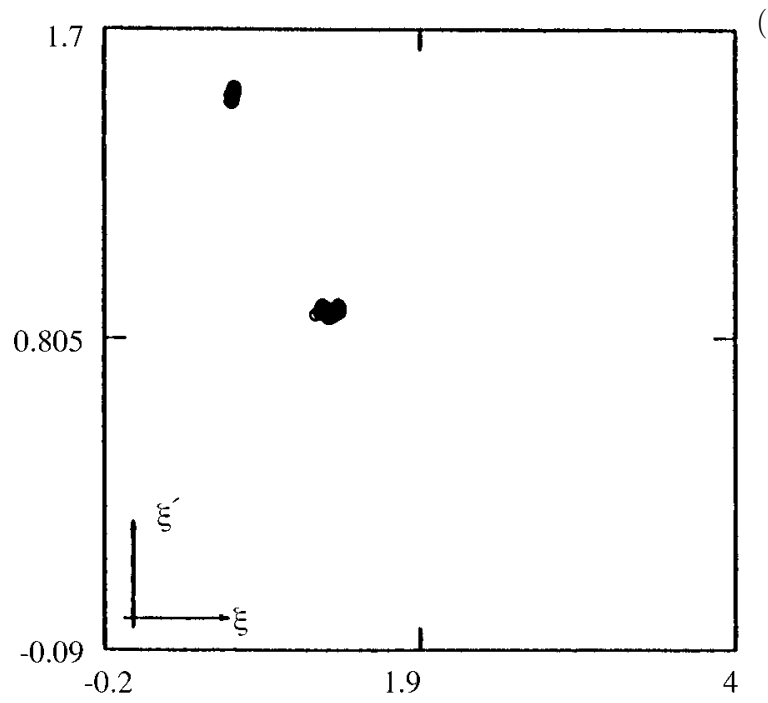

(b)

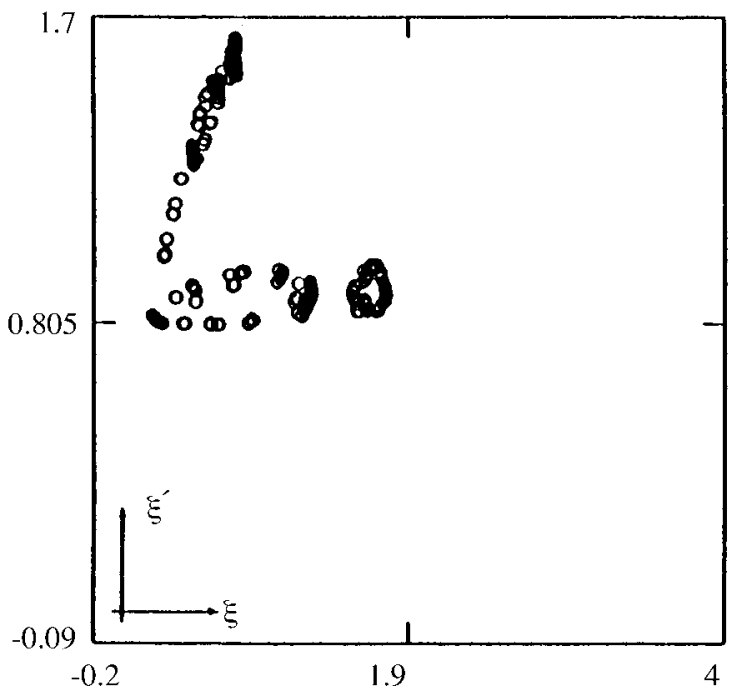

(c)

Fig. 6. Poincaré sections for $A=3.04, B=0.1$ : a) non-disturbed; b) $\varepsilon=0.5 \cdot 10^{-4}$; c) $\varepsilon=2 \cdot 10^{-4}$

case of mechanical problems with a very large number of contact points [10], this seems to be the only practicable procedure. All following results refer to the mechanical problem without physical disturbances $(\varepsilon=0)$. Fig. 8a shows an orbit for the parameter values $A=1.5$ and $B=0.1$ calculated with the constant step size $\Delta \tau=0.5$. The figure illustrates the perturbed solution for the term of 13 periods of excitation after 999 periods of transient vibration.
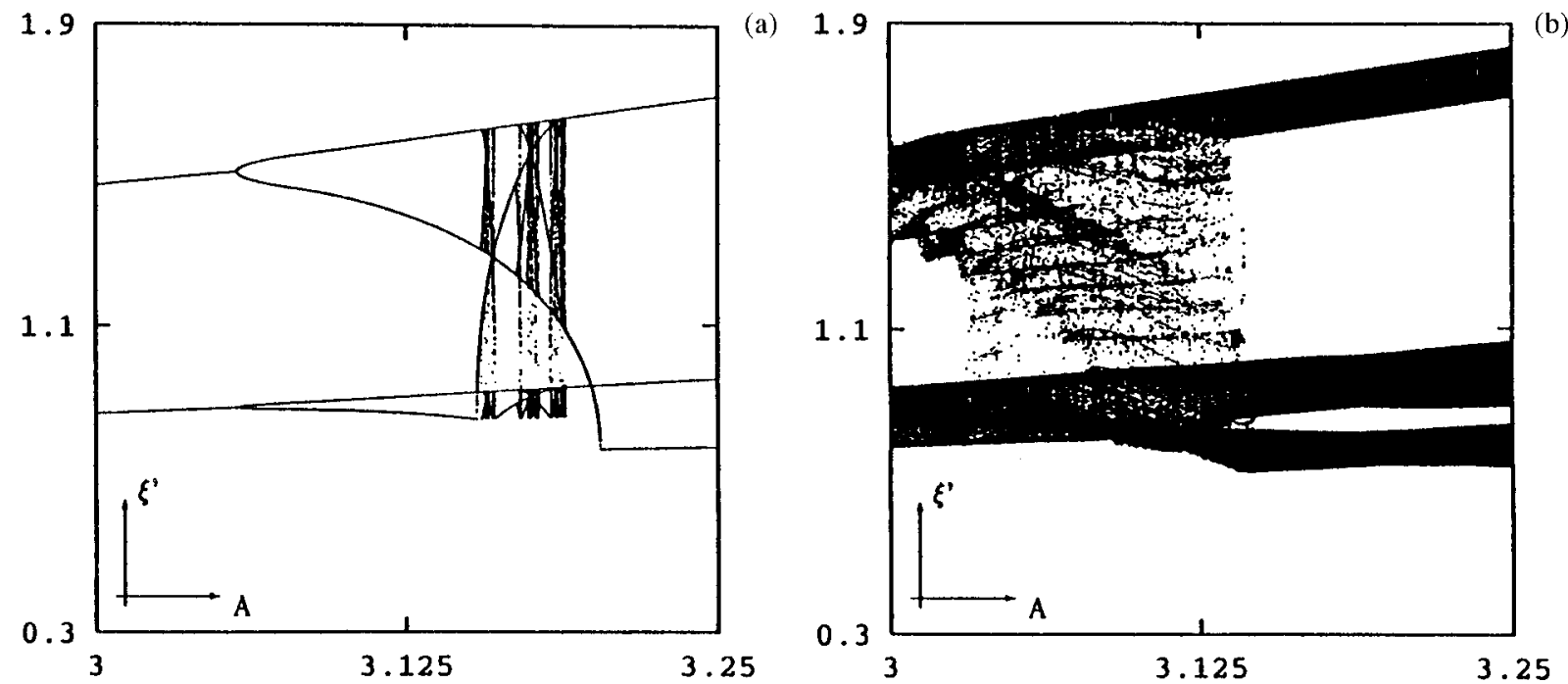

Fig. 7. Bifurcation diagram $(B=0.1)$ : a) non-disturbed; b) $\varepsilon=2 \cdot 10^{-4}$ 


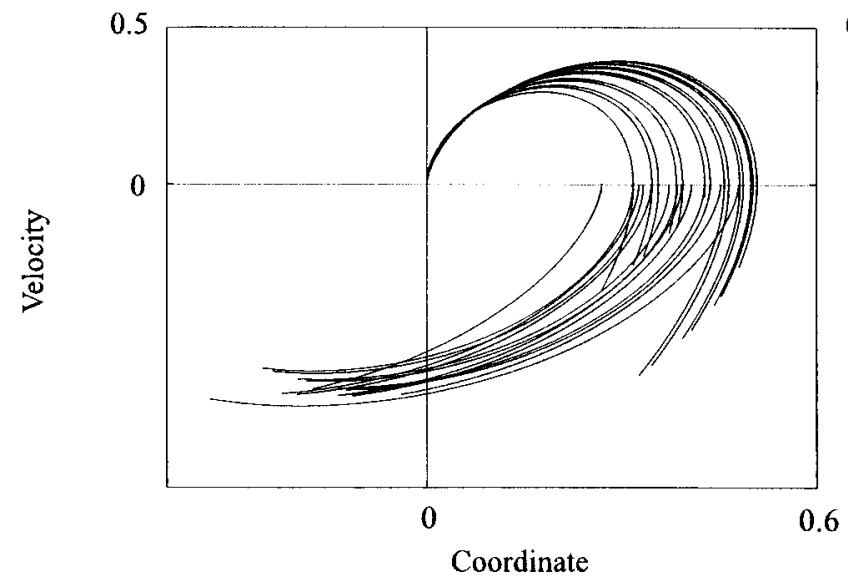

(a)

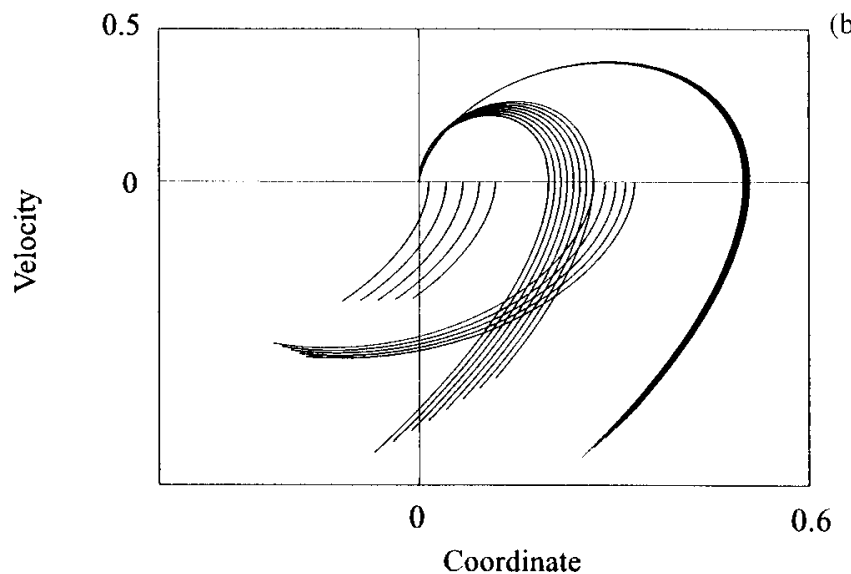

(b)

Fig. 8. Non-bifurcated solution $(A=1.5, B=0.1)$ with constant step size: a) $\Delta \tau=0.5$; b) $\Delta \tau=0.7$ without localization of the switch points

According to the definition made above, the disturbance $\Delta \tau$ under consideration is below a critical limit for practically orbital stability. Comparing Fig. 8a and Fig. 5a, we see that the structure of the orbit does not change. The phenomenon can be compared to the corresponding physical disturbances shown in Fig. 5b. However, this is valid up to a certain limit only. Fig. $8 \mathrm{~b}$ shows a further increase of the disturbance to the value $\Delta \tau=0.7$. With the exception of $\Delta \tau$ the numerical values of Fig. $8 \mathrm{a}$ were used. The orbital structure of the solution of the undisturbed problem is destroyed. Thus, the limit of the practically orbital stability has been exceeded. Due to the size of the interval $\Delta \tau$

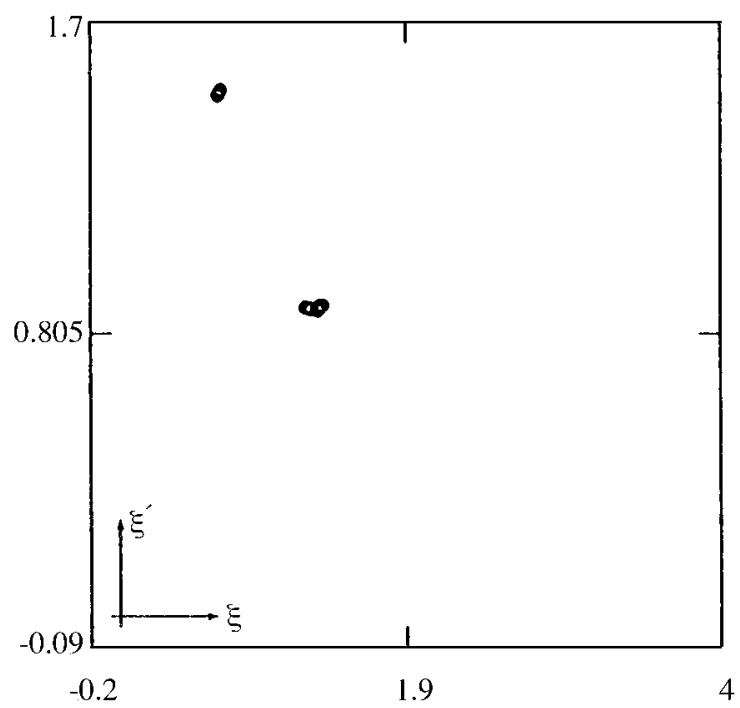

(a)
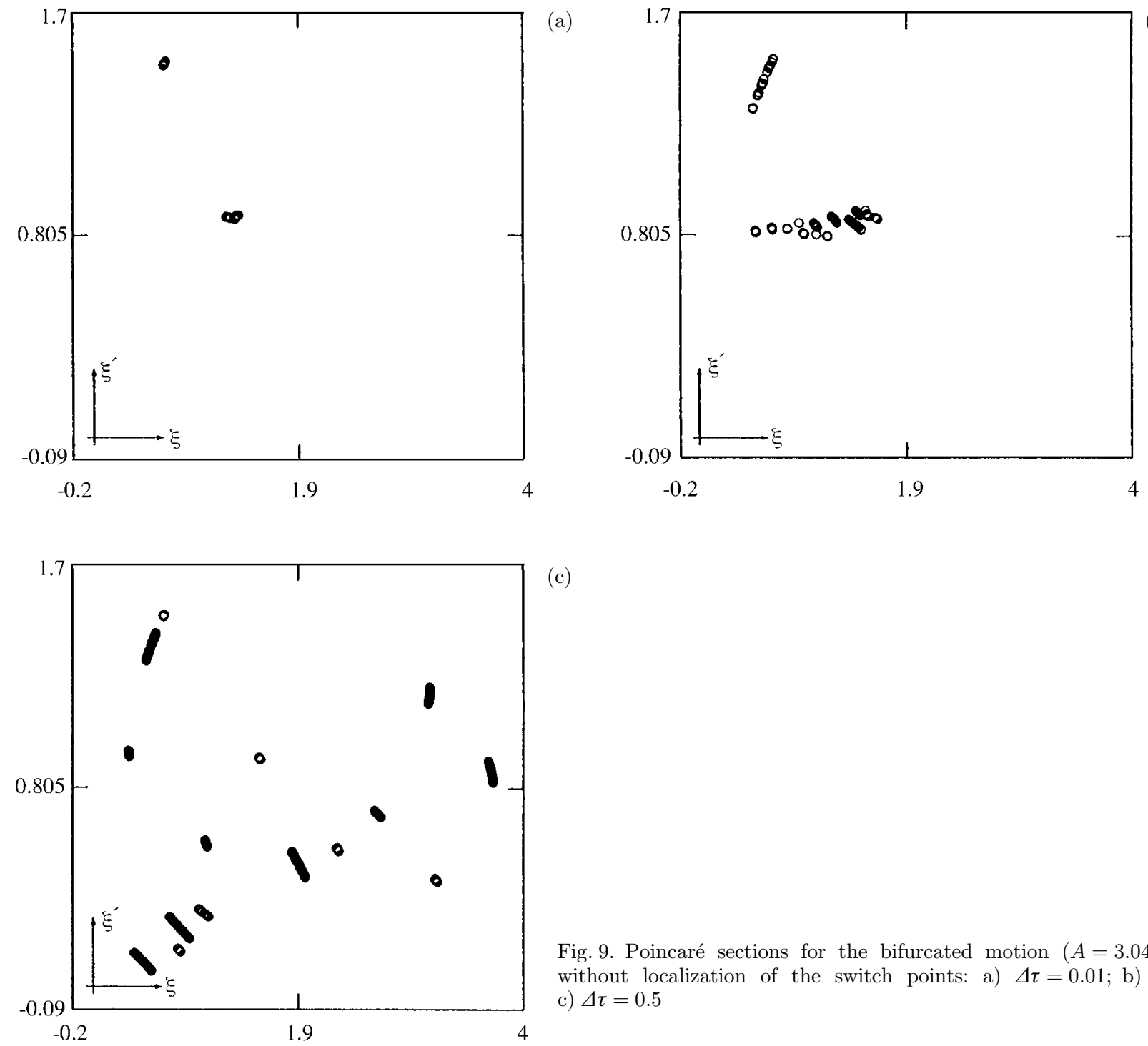

(c)

Fig. 9. Poincaré sections for the bifurcated motion $(A=3.04, B=0.1)$ without localization of the switch points: a) $\Delta \tau=0.01$; b) $\Delta \tau=0.05$; c) $\Delta \tau=0.5$ 


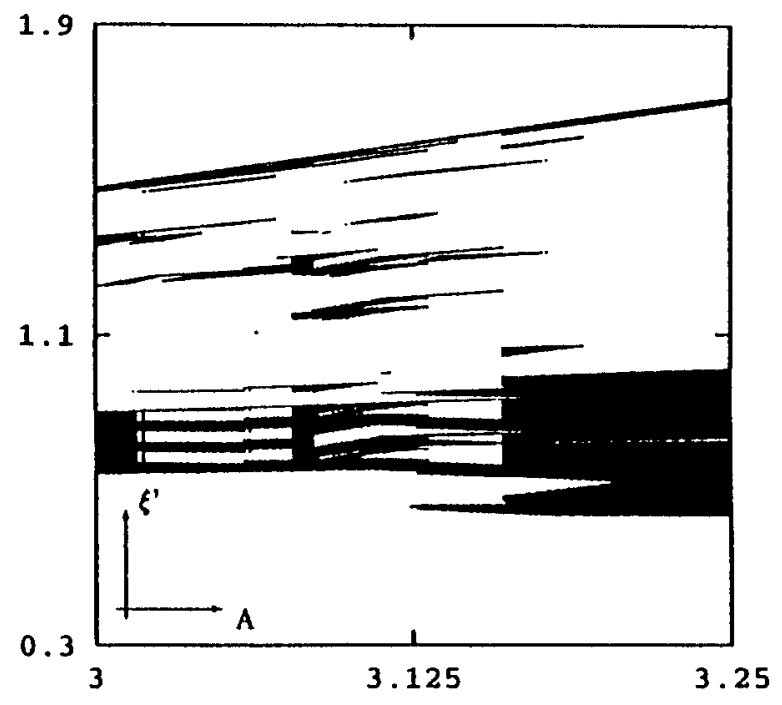

Fig. 10. Bifurcation diagram $(B=0.1)$ for numerical disturbances $\Delta \tau=0.1$

several switching points are skipped in equal intervals. This effect causes a bifurcation into a solution the period of which is a multiple of the excitation period. Therefore, it cannot be generally excluded that the solution is not only influenced by the systematic errors, but also by structural errors. These errors occur if the interval $\Delta \tau$ includes more than one zero of a switching function. If the number is even, the switching point is not recognized. If the number is odd, the wrong one is chosen. In general, this leads to drastic changes in the solution structure, since not only the switching conditions, but also the switching decision are effected.

Fig. 9 shows Poincaré sections of the bifurcated solution for $A=3.04$ and for increasing numerical disturbances. 1500 excitation periods after 1000 periods of transient vibrations are illustrated.

It is evident that comparatively small numerical disturbances (Fig. 9a and b) lead to similar results regarding orbital stability for physical disturbances. Large numerical disturbances (Fig. 9c), however, lead to a point cloud which corresponds to an irregular motion. The case $\Delta \tau=0.5$ (Fig. $9 \mathrm{~b}$ ) is near to the limit value $k_{2}$. The solution has the orbital structure of the solution of the undisturbed problem (Fig. 6a), however, the deviation from the two points can be designated as extreme. The bifurcation diagram of the comparatively large, but not yet critical, step size $\Delta \tau=0.1$ (Fig. 10) also confirms that the influences of numerical and physical disturbances are similar.

In the fifth chapter we discussed the structure of the solution of the non-disturbed problem and pointed out its non-uniqueness. Like the orbital stability, this structure has a certain limit considering the magnitude of the disturbance. This is illustrated by an example using the comparatively large constant step $\Delta \tau=0.1$ for the bifurcated solution with $A=3.04$. Fig. 11 shows the phase curves of the disturbed and the non-disturbed solutions.

It is obvious that the phase curve of the disturbed solution is much more complicated. Its structure, however, can be identified. First, different initial conditions have no effect on the stationary motion. This means that the solution has become unique. It consists of a $6 \times 2 \pi$ periodical (instead of $2 \times 2 \pi$ as for the respective non-disturbed problem) basic motion and a transient motion (duration $2 \pi$, exactly). The occurrence of the transient motion is periodical to some extent. Fig. 12 shows two basic motions and an intermediate transient motion in the form of a phase portrait and a time-displacement curve.

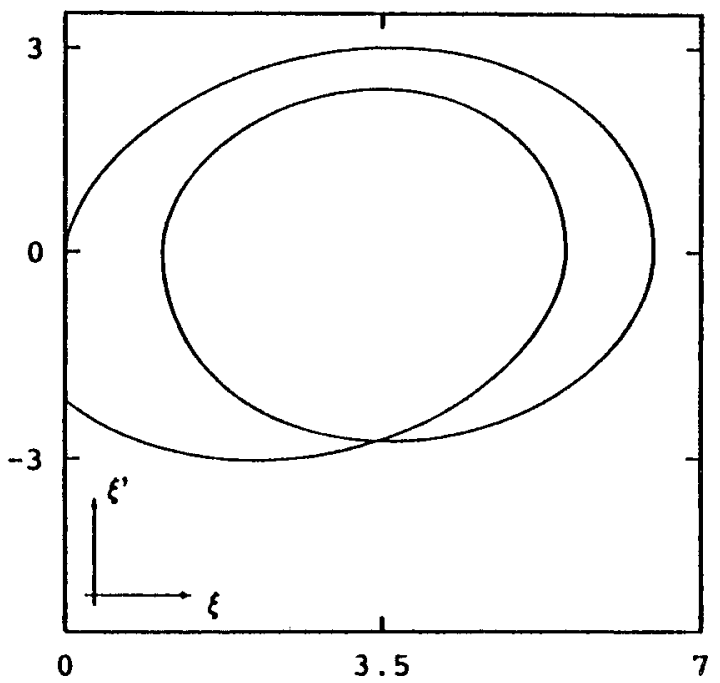

(a)

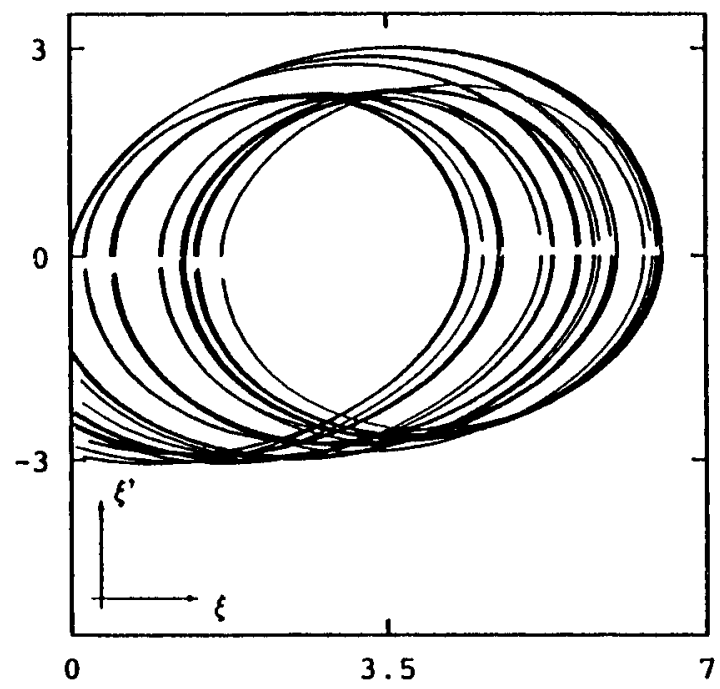

Fig. 11. Comparison of bifurcated solutions $(A=3.04, B=0.1)$ : a) exactly; b) numerical disturbances $\Delta \tau=0.1$ 


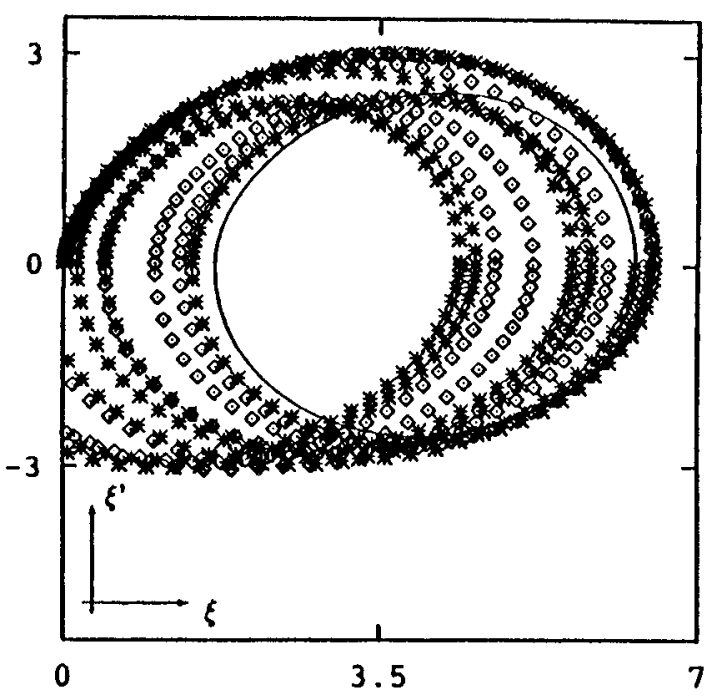

(a)

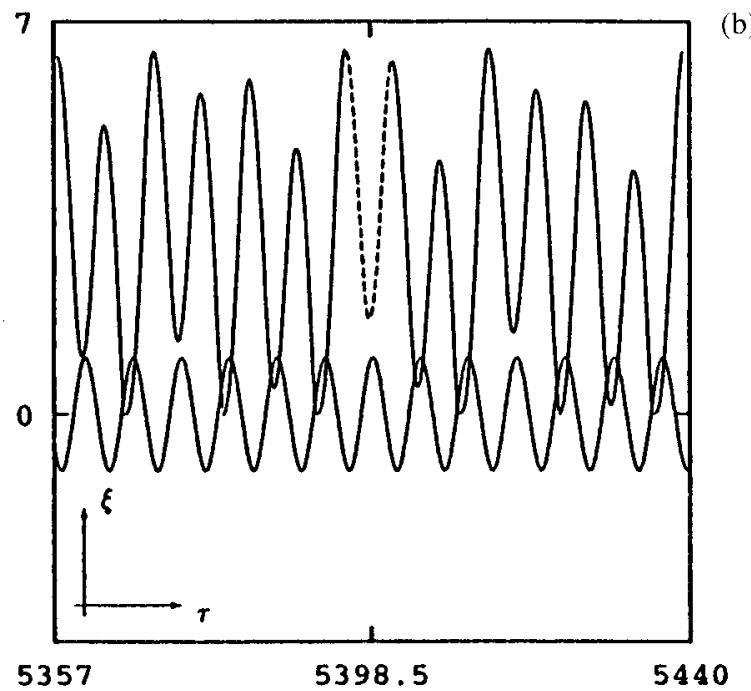

(b)

Fig. 12. Structure of the bifurcated motion $(A=3.04, B=0.1)$ in the case of numerical disturbances $(\Delta \tau=0.1)$ : a) phase curves; b) displacement-time course

In Fig. 12 the mark " $\diamond$ " denotes the basic motion before the transition, " $*$ " is the basic motion after transition and the continuous line denotes the transient motion itself. The dashed line in the time-displacement curve (Fig. 12b) clearly shows this transient motion. Fig. $12 \mathrm{~b}$ also illustrates the course of a harmonic excitation function standardized to the value one, which confirms all statements made regarding the duration of the partial motions. On the basis of the obtained structure, Fig. 11b can be commented more clearly. The illustrated phase curve consists of projections of basic motions, which vary slightly due to some inaccuracies, and the projections of a transient motion. This means that in this case we also have motions with phase differences of $2 \pi$ which, however, have a completely different kind of periodicity and are independent of the initial conditions. This example shows that the numerical calculation of non-smooth dynamic systems may lead to results which are feasible from the mechanical point of view, but are actually unrealistic.

\section{Summary}

The authors investigate a mechanical system with friction and impact with regard to stability under the influence of permanent disturbances. These disturbances are of physical nature - high frequency excitation portions - as well as of numerical nature - finite integration steps. The undisturbed problem is orbitally stable as long as the disturbances are below a critical limit. This limit is influenced by the fact that the motion is interrupted after the impact and at the point of return of the motion and due to the assumption of plastic impact. As the bifurcations increase, the interruption periods vanish completely or partly. The sensitivity to permanent disturbances increases considerably with growing bifurcations. Generally, the development of the stability scenery is similar for both types of disturbances under consideration. However, one difference is that large numerical disturbances lead to irregular system reactions, and large physical disturbances cause other types of periodicities as for the respective non-disturbed problem.

\section{References}

1 Moreau, J. J.; Panagiatopoulos, P. D. (eds.): Nonsmooth mechanics and applications. CISM, Vol. 302, Springer Verlag, Wien 1988.

2 Hahn, W.: Stability of motion. Springer-Verlag 1967.

3 Guckenheimer, J.; Holmes, P.: Nonlinear oscillations, dynamical systems, and bifurcations of vector fields. Springer-Verlag 1983.

4 Verspohl, J.: Ungefesselte hysteretische Systeme unter besonderer Berücksichtigung der Vibrationsrammung. Diss. Univ. Karlsruhe 1990.

5 Storz, M.: Stabilität der Bewegung eines Reibschwingers mit Stoß am Beispiel des Vibrationsrammens. Diss. Univ. Karlsruhe 1994.

6 EICH, E.: Projizierende Mehrschrittverfahren zur numerischen Lösung von Bewegungsgleichungen technischer Mehrkörpersysteme mit Zwangsbedingungen und Unstetigkeiten. Fortschritt-Berichte VDI, Reihe 18, Nr. 109, VDI-Verlag, Düsseldorf 1992.

7 Reithmeyer, E.: Periodic solutions of nonlinear dynamical systems with discontinuities. In: SchieHLEn, W. (ed.): Nonlinear dynamics in engineering systems. Springer-Verlag 1990.

8 MeIJAard, J. P.: Efficient numerical integration of the equations of motion of non-smooth mechanical systems. ZAMM 77 (1997) $6,419-427$.

9 Muller, D. E.: A method for solving algebraic equations using an automatic computer. Math. Tables Aids-Comput. 10 (1956), $208-215$.

10 Jean, M.; Moreau, J. J.: Unilaterality and dry friction in the dynamics of rigid body collections. Lab. Méc. Genie Civil, Univ. Montpellier II, Preprint Nr. 92-2, 1992.

Received October 24, 1997, revised July 27, 1998, accepted August 24, 1998

Address: Prof. Dr.-Ing. habil. Peter Vielsack; Dr. Andreas Hartung, Institut für Mechanik, Universität Karlsruhe, Kaiserstr. 12 , D-76131 Karlsruhe, Germany 